Swarthmore College

Works

$2-1-2012$

\title{
Generalized Eigenproblem And Nonlinear Elliptic Equations With Nonlinear Boundary Conditions
}

\author{
Nsoki Mavinga \\ Swarthmore College, nmaving1@swarthmore.edu
}

Follow this and additional works at: https://works.swarthmore.edu/fac-math-stat

Part of the Mathematics Commons

Let us know how access to these works benefits you

\section{Recommended Citation}

Nsoki Mavinga. (2012). "Generalized Eigenproblem And Nonlinear Elliptic Equations With Nonlinear Boundary Conditions". Proceedings Of The Royal Society Of Edinburgh, Section A: Mathematics. Volume 142, Issue 1. 137-153. DOI: 10.1017/S0308210510000065

https://works.swarthmore.edu/fac-math-stat/78

This work is brought to you for free by Swarthmore College Libraries' Works. It has been accepted for inclusion in Mathematics \& Statistics Faculty Works by an authorized administrator of Works. For more information, please contact myworks@swarthmore.edu. 


\title{
Generalized eigenproblem and nonlinear elliptic equations with nonlinear boundary conditions
}

\author{
N. Mavinga* \\ Department of Mathematics, University of Rochester, Rochester, \\ NY 14627-0138, USA (mavinga@math.rochester.edu)
}

(MS received 8 January 2010; accepted 22 March 2011)

\begin{abstract}
We are concerned with the solvability of nonlinear second-order elliptic partial differential equations with nonlinear boundary conditions. We study the generalized Steklov-Robin eigenproblem (with possibly singular weights) in which the spectral parameter is both in the differential equation and on the boundary. We prove the existence of solutions for nonlinear problems when both nonlinearities in the differential equation and on the boundary interact, in some sense, with the generalized spectrum. The proofs are based on variational methods and a priori estimates.
\end{abstract}

\section{Introduction}

We are concerned with the existence of (weak) solutions to the following nonlinear elliptic boundary-value problem:

$$
\left.\begin{array}{rl}
-\Delta u+c(x) u=f(x, u) & \text { in } \Omega, \\
\frac{\partial u}{\partial \nu}+\sigma(x) u=g(x, u) & \text { on } \partial \Omega,
\end{array}\right\}
$$

where the nonlinear reaction-function $f(x, u)$ and the nonlinearity on the boundary $g(x, u)$ interact, in some sense, with the generalized Steklov-Robin spectrum of the following linear problem (with possibly singular $(m, \rho)$-weights)

$$
\left.\begin{array}{cc}
-\Delta u+c(x) u=\mu m(x) u & \text { in } \Omega, \\
\frac{\partial u}{\partial \nu}+\sigma(x) u=\mu \rho(x) u & \text { on } \partial \Omega .
\end{array}\right\}
$$

Unlike previous results in the literature, what sets our results apart is that we compare both the reaction nonlinearity $f$ in the differential and the boundary nonlinearity $g$ with higher eigenvalues of the spectrum of problem (1.2), which we describe herein (for the first time, to the best of our knowledge), where the spectral parameter is both in the differential equation and on the boundary (with weights). Several

* Present address: Department of Mathematics and Statistics, Swarthmore College, Swarthmore, PA 19081-1390, USA (mavinga@swarthmore.edu).

(C) 2012 The Royal Society of Edinburgh 
works have been devoted to the study of the solvability of the elliptic boundaryvalue problem (1.1) and (1.2). We refer the interested reader to $[1,2,4,8,9,11,12,18]$ and the references therein.

Concerning the eigenproblem (1.2), results have been obtained by many authors. We mention the paper by Steklov [23], who initiated the problem (with $m \equiv 0$ ) on a disc in 1902, then Bandle [3] and Auchmuty [2] extended Steklov's results to higher dimensions. We refer also to Amann [1] who discussed only the existence of the first eigenvalue of the spectrum for this problem under somewhat strong regularity conditions on the data. We shall show the existence of the entire generalized Steklov-Robin spectrum for problem (1.2). This spectrum includes the Steklov, Neumann and Robin spectra. The present approach differs from that carried out in $[1,2]$. The arguments in [2] involved maximization of the boundary functional on bounded closed convex subsets of $H^{1}(\Omega)$. In [1], the techniques used the theory of positive operators (Krein-Rutman theorem), which, of course, does not apply when trying to obtain higher eigenvalues. Here we use the minimization of the (energy) functional on an appropriate subspace of $H^{1}(\Omega)$, which is derived herein. We also discuss some properties of the spectrum, such as the principality of the least positive eigenvalue of this problem, which was not treated in [2]. Our results generalize those recently obtained in [2], where only the case in which $m \equiv 0$ and $\rho \not \equiv 0$ was discussed, and the principality of the first eigenvalue was not treated. Moreover, they extend to the existence of higher eigenvalues a result obtained in [1], where only the existence of the first eigenvalue is discussed.

Concerning the nonlinear problem (1.1), there are some (scattered) existence results in the literature; we refer the reader to $[1,17]$ and references therein. To the best of our knowledge, little work has been carried out on this problem, especially in the framework of the generalized Steklov-Robin spectrum that we describe here. A few results on a disc $(n=2)$ were obtained in the case of the Steklov spectrum by Cushing [6] and Klingelhöfer [13]. (The results in [13] were significantly generalized to higher dimensions in [1] in the framework of sub- and super-solutions method as aforementioned.) We also refer to Klingelhöfer [14], where monotonicity methods were used for nonlinearities near the first eigenvalue. Recently, Nkashama and Mavinga [17] proved the non-resonance results for problem (1.1) in which the nonlinearities interact, in some sense, only with either the Steklov or the Neumann spectrum. It is our purpose in this paper to prove the existence results for problem (1.1) in which both nonlinearities in the differential equation and on the boundary interact, in some sense, with the generalized Steklov-Robin spectrum. Of particular interest are the non-resonance results below the first (generalized) Steklov-Robin eigenvalue as well as between two consecutive (generalized) SteklovRobin eigenvalues.

This paper is organized as follows. Section 2 is devoted to the generalized SteklovRobin eigenproblem (1.2). In $\S 3$ the existence results for the nonlinear problem (1.1) are stated. These results are proved in $\S 4$. We conclude the paper with some remarks that show (among other things) how our results can be extended to problems with variable coefficients.

Throughout the paper we will assume that $\Omega$ is a bounded domain in $\mathbb{R}^{n}, n \geqslant 2$, with boundary $\partial \Omega$ of class $C^{0,1}, \partial / \partial \nu$ is the (unit) outward normal derivative on $\partial \Omega$. Note that the eigenproblem (1.2) includes as special cases the weighted Steklov 
eigenproblem (when $m \equiv 0$ and $\rho \not \equiv 0$ ) that was considered in $[2,3,23]$ as well as the weighted Robin-Neumann eigenproblem (when $\rho \equiv 0$ and $m \not \equiv 0$ ); the latter is also referred to in the literature as the Neumann or regular oblique derivative boundary condition (see, for example, $[1,7,16]$ and the references therein).

By a weak solution of equation (1.1), we mean a function $u \in H^{1}(\Omega)$ such that

$$
\int \nabla u \nabla v+\int c(x) u v+\oint \sigma(x) u v=\int f(x, u) v+\oint g(x, u) v \quad \text { for all } v \in H^{1}(\Omega) .
$$

Throughout this paper, $H^{1}(\Omega)$ denotes the usual real Sobolev space of functions on $\Omega$ endowed with the $(c, \sigma)$-inner product defined as

$$
(u, v)_{(c, \sigma)}=\int \nabla u \nabla v+\int c(x) u v+\oint \sigma(x) u v
$$

with the associated norm denoted by $\|u\|_{(c, \sigma)}$. This norm is equivalent to the standard $H^{1}(\Omega)$-norm.

The functions $c: \Omega \rightarrow \mathbb{R}, \sigma: \partial \Omega \rightarrow \mathbb{R}, f: \bar{\Omega} \times \mathbb{R} \rightarrow \mathbb{R}$ and $g: \bar{\Omega} \times \mathbb{R} \rightarrow \mathbb{R}$ satisfy the following conditions.

$\left(\mathrm{C}_{1}\right) c \in L^{p}(\Omega)$ with $p \geqslant \frac{1}{2} n$ when $n \geqslant 3(p>1$ when $n=2)$ and $\sigma \in L^{q}(\partial \Omega)$ with $q \geqslant n-1$ when $n \geqslant 3(q>1$ when $n=2)$ with $(c, \sigma)>0$; that is,

$$
c(x) \geqslant 0 \quad \text { almost everywhere (a.e.) on } \Omega
$$

and

$$
\sigma(x) \geqslant 0 \quad \text { a.e. on } \partial \Omega \text { such that } \int c(x) \mathrm{d} x+\oint \sigma(x) \mathrm{d} x>0,
$$

where, throughout this paper, $\int$ denotes the (volume) integral on $\Omega$ and $\oint$ denotes the (surface) integral on $\partial \Omega$.

$\left(\mathrm{C}_{2}\right) f \in C(\bar{\Omega} \times \mathbb{R})$ and $g \in C(\bar{\Omega} \times \mathbb{R})$.

$\left(\mathrm{C}_{3}\right)$ There exist constants $a_{1}, a_{2}>0$ such that

$$
|g(x, u)| \leqslant a_{1}+a_{2}|u|^{s} \quad \text { with } 0 \leqslant s<\frac{n}{n-2} .
$$

$\left(\mathrm{C}_{3^{\prime}}\right)$ There exist constants $b_{1}, b_{2}>0$ such that

$$
|f(x, u)| \leqslant b_{1}+b_{2}|u|^{s} \quad \text { with } 0 \leqslant s<\frac{n+2}{n-2} .
$$

\section{Generalized Steklov-Robin eigenproblems}

In this section we will first study the generalized spectrum that will be used for the comparison with the nonlinearities in equation (1.1). This spectrum includes the Steklov, Neumann and Robin spectra. We therefore generalize the results in $[1-3,23]$. 
Consider the linear problem

$$
\left.\begin{array}{rr}
-\Delta u+c(x) u=\mu m(x) u & \text { in } \Omega, \\
\frac{\partial u}{\partial \nu}+\sigma(x) u=\mu \rho(x) u & \text { on } \partial \Omega
\end{array}\right\}
$$

where $(m, \rho) \in L^{p}(\Omega) \times L^{q}(\partial \Omega)$ with $p \geqslant \frac{1}{2} n$ and $q \geqslant n-1$ when $n \geqslant 3(p, q>1$ when $n=2)$ and $(m, \rho)>0$; that is,

$$
\left.\begin{array}{ll}
m(x) \geqslant 0 & \text { a.e. on } \Omega, \\
\rho(x) \geqslant 0 & \text { a.e. on } \partial \Omega \text { such that } \int m(x) \mathrm{d} x+\oint \rho(x) \mathrm{d} x>0 .
\end{array}\right\}
$$

(We stress the fact that the weight functions $m$ and $\rho$ may vanish on subsets of positive measure.)

The (generalized) Steklov-Robin eigenproblem is to find a pair $(\mu, \varphi) \in \mathbb{R} \times$ $H^{1}(\Omega)$ with $\varphi \not \equiv 0$ such that

$$
\begin{aligned}
& \int \nabla \varphi \nabla v+\int c(x) \varphi v+\oint \sigma(x) \varphi v \\
&=\mu\left(\int m(x) \varphi v+\oint \rho(x) \varphi v\right) \text { for all } v \in H^{1}(\Omega) .
\end{aligned}
$$

Picking $v=\varphi$, we see that, if there is such an eigenpair, then

$$
\mu>0 \text { and } \int m(x) \varphi^{2}+\oint \rho(x) \varphi^{2}>0 .
$$

(Otherwise, $\varphi$ would be a constant function; which would contradict the assumptions imposed on $c(x)$ and $\sigma(x)$. Note that if $c \equiv 0$ and $\sigma \equiv 0$, then $\mu=0$ is an eigenvalue of equation (2.1) with eigenfunction $\varphi \equiv 1$ on $\bar{\Omega}$.) It is therefore appropriate to consider the closed linear subspace of $H^{1}(\Omega)$ defined by

$$
V_{(m, \rho)}(\Omega):=\left\{u \in H^{1}(\Omega): \int m(x) u^{2}+\oint \rho(x) u^{2}=0\right\}
$$

and to look for the eigenfunctions associated with equation $(2.1)$ in the $(c, \sigma)$ orthogonal complement $\left[V_{(m, \rho)}(\Omega)\right]^{\perp}=H_{(m, \rho)}^{1}(\Omega)$ of this subspace in $H^{1}(\Omega)$. Note that if we set $\Omega(m):=\{x \in \Omega: m(x)>0\}$ and $\partial \Omega(\rho):=\{x \in \partial \Omega: \rho(x)>0\}$, then

$$
V_{(m, \rho)}(\Omega)=\left\{u \in H^{1}(\Omega): u=0 \text { a.e. in } \Omega(m) \text { and } \Gamma u=0 \text { a.e. in } \partial \Omega(\rho)\right\},
$$

where $\Gamma u$ denotes the trace of $u$ on $\partial \Omega$. Observe that if $m \equiv 0$ in $\Omega$ and $\rho(x)>0$ a.e. on $\partial \Omega$, then the subspace $V_{(m, \rho)}(\Omega)$ reduces simply to $H_{0}^{1}(\Omega)$, whereas, for $m(x)>0$ a.e. in $\Omega$ and $\rho \equiv 0$ on $\partial \Omega, V_{(m, \rho)}(\Omega)$ reduces to the trivial set $\{0\}$. Thus, one can split the Hilbert space $H^{1}(\Omega)$ as a direct $(c, \sigma)$-orthogonal sum in the following way:

$$
H^{1}(\Omega)=V_{(m, \rho)}(\Omega) \oplus H_{(m, \rho)}^{1}(\Omega) .
$$


Note also that if $(\mu, \varphi) \in \mathbb{R} \times H^{1}(\Omega)$ is an eigenpair, then it follows from the definition of $V_{(m, \rho)}(\Omega)$ that

$$
\int \nabla \varphi \nabla v+\int c(x) \varphi v+\oint \sigma(x) \varphi v=0 \quad \text { for all } v \in V_{(m, \rho)}(\Omega)
$$

that is, $\varphi \in H_{(m, \rho)}^{1}(\Omega)$.

Besides the Sobolev space $H^{1}(\Omega)$, we shall make use in what follows of the real Lebesgue spaces $L^{q}(\partial \Omega)$ for $1 \leqslant q \leqslant \infty$, and of the continuity and compactness of the trace operator

$$
\Gamma: H^{1}(\Omega) \rightarrow L^{q}(\partial \Omega) \quad \text { for } 1 \leqslant q<\frac{2(n-1)}{n-2}
$$

(see, for example, $[15,19]$ and the references therein). Sometimes we will just use $u$ in place of $\Gamma u$ when considering the trace of a function on $\partial \Omega$. Throughout this paper we denote the $L^{2}(\partial \Omega)$-inner product by $(u, v)_{\partial}=\oint u v$ and the associated norm by $\|u\|_{\partial}$. We also set

$$
(u, v)_{(m, \rho)}:=\int m(x) u v+\oint \rho(x) u v \text { and }\|u\|_{(m, \rho)}^{2}:=\int m(x) u^{2}+\oint \rho(x) u^{2}
$$

for $u, v \in H^{1}(\Omega)$.

Using the Hölder inequality, the continuity of the trace operator, the Sobolev embedding theorem and the lower semicontinuity of $\|\cdot\|(c, \sigma)$, we deduce that $\|\cdot\|_{(c, \sigma)}$ (see, for example, (1.4)) is equivalent to the standard $H^{1}(\Omega)$-norm. This observation enables us to prove the existence of an unbounded and discrete spectrum for the Steklov-Robin eigenproblem (2.1), and discuss some of its properties.

TheOREM 2.1. Assume that $c$, $\sigma$ satisfy condition $\left(\mathrm{C}_{1}\right)$ and that $(m, \rho) \in L^{p}(\Omega) \times$ $L^{q}(\partial \Omega)$ with $(m, \rho)>0$ (see (2.2)). Then we have the following.

(i) The Steklov-Robin eigenproblem (2.1) has a sequence of real eigenvalues

$$
0<\mu_{1}<\mu_{2} \leqslant \cdots \leqslant \mu_{j} \leqslant \cdots \rightarrow \infty \text { as } j \rightarrow \infty,
$$

each eigenvalue has a finite-dimensional eigenspace.

(ii) The eigenfunctions $\varphi_{j}$ corresponding to the eigenvalues $\mu_{j}$ form an $(c, \sigma)$ orthogonal and $(m, \rho)$-orthonormal family in $H_{(m, \rho)}^{1}(\Omega)$ (a closed linear subspace of $\left.H^{1}(\Omega)\right)$.

(iii) The normalized eigenfunctions provide a complete $(c, \sigma)$-orthonormal basis of $H_{(m, \rho)}^{1}(\Omega)$. Moreover, each function $u \in H_{(m, \rho)}^{1}(\Omega)$ has a unique representation of the form

$$
\left.\begin{array}{c}
u=\sum_{j=1}^{\infty} c_{j} \varphi_{j} \quad \text { with } c_{j}:=\frac{1}{\mu_{j}}\left(u, \varphi_{j}\right)_{(c, \sigma)}=\left(u, \varphi_{j}\right)_{(m, \rho)}, \\
\|u\|_{(c, \sigma)}^{2}=\sum_{j=1}^{\infty} \mu_{j}\left|c_{j}\right|^{2}
\end{array}\right\}
$$


In addition,

$$
\|u\|_{(m, \rho)}^{2}=\sum_{j=1}^{\infty}\left|c_{j}\right|^{2}
$$

Proof. 1. We wish to prove the existence of a sequence of real eigenvalues $\left\{\mu_{j}\right\}$ and the eigenfunctions $\varphi_{j}$ corresponding to the eigenvalues $\mu_{j}$ that form an orthogonal family in $H_{(m, \rho)}^{1}(\Omega)$.

Let us define the functionals

$$
\left.\begin{array}{l}
P(u)=\int|\nabla u|^{2}+\int c(x) u^{2}+\oint \sigma(x) u^{2}, \\
Q(u)=\int m(x) u^{2}+\oint \rho(x) u^{2}-1
\end{array}\right\}
$$

for $u \in H^{1}(\Omega) . P$ and $Q$ are $C^{1}$-functional with

$$
\left.\begin{array}{l}
P^{\prime}(u) v=2\left[\int \nabla u \nabla v+\int c(x) u v+\oint \sigma(x) u v\right], \\
Q^{\prime}(u) v=2\left[\int m(x) u v+\oint \rho(x) u v\right]
\end{array}\right\}
$$

for $u, v \in H^{1}(\Omega)$. Now we shall prove that $P$ attains its minimum on the constraint set $W=\left\{u \in H_{(m, \rho)}^{1}(\Omega): Q(u)=0\right\}$. Let $\eta=\inf _{u \in W} P(u)$. Then, using the continuity of the trace operator, the Sobolev embedding theorem and the lower semicontinuity of $\|\cdot\|_{(c, \sigma)}$, one can show that there exists $\varphi_{1}$ such that $P\left(\varphi_{1}\right)=\eta$. Hence, $P$ attains its minimum at $\varphi_{1}$ and $\varphi_{1}$ satisfies the Euler-Lagrange equation

$$
\int \nabla \varphi_{1} \nabla v+\int c(x) \varphi_{1} v+\oint \sigma(x) \varphi_{1} v=\mu_{1}\left(\int m(x) \varphi_{1} v+\oint \rho(x) \varphi_{1} v\right)
$$

for the Lagrange multiplier $\mu_{1}$ and for all $v \in H^{1}(\Omega)$. We see that $\left(\mu_{1}, \varphi_{1}\right)$ satisfies (2.3) and $\varphi_{1} \in H_{(m, \rho)}^{1}(\Omega)$. If we take $v=\varphi_{1}$ in $(2.11)$, we obtain that the eigenvalue $\mu_{1}$ is the infimum $\eta=P\left(\varphi_{1}\right)=\mu_{1}$. This means that we could define $\mu_{1}$ by the Rayleigh quotient

$$
\mu_{1}=\inf _{\substack{u \in H^{1} \\ u \neq 0}} \frac{\|u\|_{(c, \sigma)}^{2}}{\int m(x) u^{2}+\oint \rho(x) u^{2}} .
$$

Clearly, $\mu_{1}=P\left(\varphi_{1}\right) \geqslant 0$. In fact, if $P\left(\varphi_{1}\right)=0$, then $\left|\nabla \varphi_{1}\right|=0$ on $\Omega$, hence $\varphi_{1}$ must be a constant that contradicts the assumptions imposed on $c(x)$ and $\sigma(x)$. Thus, $\mu_{1}>0$.

Now we shall prove the existence of higher eigenvalues. Define

$$
W_{1}=\left\{u \in H_{(m, \rho)}^{1}(\Omega):\left(u, \varphi_{1}\right)_{(m, \rho)}=0\right\} .
$$

Since $W_{1}$ is the nullspace of the continuous functional $\left(\cdot, \varphi_{1}\right)_{(m, \rho)}$ on $H_{(m, \rho)}^{1}(\Omega), W_{1}$ is a closed subspace of $H_{(m, \rho)}^{1}(\Omega)$, and it is therefore a Hilbert space itself under the same inner product $(\cdot, \cdot)_{(c, \sigma)}$. We can now define

$$
\mu_{2}=\inf \left\{P(u): u \in W_{1} \text { and }\|u\|_{(m, \rho)}=1\right\}=\inf _{\substack{u \in W_{1} \\ u \neq 0}} \frac{\|u\|_{(c, \sigma)}^{2}}{\int m(x) u^{2}+\oint \rho(x) u^{2}},
$$


since $W_{1} \subset W$, one has $\mu_{1} \leqslant \mu_{2}$. Moreover, we can repeat the above arguments to show that $\mu_{2}$ is achieved at some $\varphi_{2} \in H_{(m, \rho)}^{1}(\Omega)$.

Proceeding inductively, we let

$$
W_{j}=\left\{u \in H_{(m, \rho)}^{1}(\Omega):\left(u, \varphi_{i}\right)_{(m, \rho)}=0 \text { for } i=1, \ldots, j\right\}
$$

and

$$
\mu_{j+1}=\inf \left\{P(u): u \in W_{j} \text { and }\|u\|_{(m, \rho)}=1\right\}=\inf _{\substack{u \in W_{j} \\ u \neq 0}} \frac{\|u\|_{(c, \sigma)}^{2}}{\int m(x) u^{2}+\oint \rho(x) u^{2}} .
$$

In this way, we generate a sequence of eigenvalues

$$
0<\mu_{1} \leqslant \mu_{2} \leqslant \cdots \leqslant \mu_{j} \leqslant \cdots
$$

whose associated $\varphi_{j}$ are $(c, \sigma)$-orthogonal and $(m, \rho)$-orthonormal in $H_{(m, \rho)}^{1}(\Omega)$.

2 . We wish to prove that $\mu_{j} \rightarrow \infty$ as $j \rightarrow \infty$ and each eigenvalue $\mu_{j}$ has a finitedimensional eigenspace. Suppose by contradiction that the sequence $\mu_{j}$ is bounded above by a constant. Therefore, the corresponding sequence of eigenfunctions $\varphi_{j}$ is bounded in $H^{1}(\Omega)$. By the Rellich-Kondrachov theorem and the compactness of the trace operator, there is a Cauchy subsequence (which we again denote by $\varphi_{j}$ ) such that

$$
\left\|\varphi_{j}-\varphi_{k}\right\|_{(m, \rho)}^{2} \rightarrow 0
$$

Since the $\varphi_{j}$ are $(m, \rho)$-orthonormal, we get that

$\left\|\varphi_{j}-\varphi_{k}\right\|_{(m, \rho)}^{2}=\left\|\varphi_{j}\right\|_{(m, \rho)}^{2}+\left\|\varphi_{k}\right\|_{(m, \rho)}^{2}=2>0$ if $j \neq k$, which contradicts (2.16).

Thus, $\mu_{j}$ must tend to $\infty$. As a consequence of $\mu_{j} \rightarrow \infty$, we have that each $\mu_{j}$ occurs only finitely many times.

3 . We wish to prove that the normalized eigenfunctions provide a complete orthonormal basis of $H_{(m, \rho)}^{1}(\Omega)$. Let $\psi_{j}=1 / \sqrt{\mu_{j}} \varphi_{j}$, so that $\left\|\psi_{j}\right\|_{(c, \sigma)}=1$. We shall prove that the sequence $\left\{\psi_{j}\right\}$ is a maximal $(c, \sigma)$-orthonormal family of $H_{(m, \rho)}^{1}(\Omega)$. Suppose by contradiction that the sequence $\left\{\psi_{j}\right\}$ is not maximal. Then there exists a $\xi \in H_{(m, \rho)}^{1}(\Omega)$ such that $\|\xi\|_{(c, \sigma)}=1$ and $\left(\xi, \psi_{j}\right)_{(c, \sigma)}=0$. Therefore, $\left(\xi, \psi_{j}\right)_{(m, \rho)}=$ 0 . We have that $\xi \in W_{j}$ for all $j \geqslant 1$. It follows from the definition of $\mu_{j}$ in (2.14) that

$$
\mu_{j}\left(\int m(x) \xi^{2}+\oint \rho(x) \xi^{2}\right) \leqslant\|\xi\|_{(c, \sigma)}^{2}=1 \text { for all } j \geqslant 1 .
$$

Since $\mu_{j} \rightarrow \infty$, we get that $\|\xi\|_{(m, \rho)}=0$. Hence, $\xi=0$, which contradicts the definition of $\xi$. Thus, the sequence $\left\{\psi_{j}\right\}$ provides a complete orthonormal basis of $H_{(m, \rho)}^{1}(\Omega) ;$ that is, for any $u \in H_{(m, \rho)}^{1}(\Omega)$,

$$
\begin{gathered}
u=\sum_{j=1}^{\infty} \alpha_{j} \psi_{j} \quad \text { with } \alpha_{j}=\left(u, \psi_{j}\right)_{(c, \sigma)}, \\
\|u\|_{(c, \sigma)}^{2}=\sum_{j=1}^{\infty}\left|\alpha_{j}\right|^{2} .
\end{gathered}
$$


Therefore,

$$
u=\sum_{j=1}^{\infty} c_{j} \varphi_{j} \quad \text { and } \quad\|u\|_{(c, \sigma)}^{2}=\sum_{j=1}^{\infty} \mu_{j}\left|c_{j}\right|^{2}
$$

with

$$
c_{j}=\frac{1}{\mu_{j}\left(u, \varphi_{j}\right)_{(c, \sigma)}}=\left(u, \varphi_{j}\right)_{(m, \rho)} .
$$

Now we shall show that

$$
\|u\|_{(m, \rho)}^{2}=\sum_{j=1}^{\infty}\left|c_{j}\right|^{2} .
$$

Indeed, from the characterization of $\mu_{1}$ in (2.12) and (2.17), we have that

$$
\left\|u-\sum_{j=1}^{k} c_{j} \varphi_{j}\right\|_{(m, \rho)}^{2} \rightarrow 0 \quad \text { as } k \rightarrow \infty .
$$

Then, by the continuity of $\|\cdot\|_{(m, \rho)}$ and the $(m, \rho)$-orthonormality of $\varphi_{j}$, we get that

$$
\|u\|_{(m, \rho)}^{2}=\sum_{j=1}^{\infty}\left|c_{j}\right|^{2} .
$$

The proof is complete.

The following result gives a variational characterization of the eigenvalues and a splitting of the space $H_{(m, \rho)}^{1}(\Omega)$ (and, hence, of $H^{1}(\Omega)$ ) which will be needed in the proofs of the results on nonlinear problems.

Corollary 2.2. Assume that $c$ and $\sigma$ satisfy condition $\left(\mathrm{C}_{1}\right)$ and $(m, \rho) \in L^{p}(\Omega) \times$ $L^{q}(\partial \Omega)$ with $(m, \rho)>0$ (see (2.2)). Then we have the following.

(i) For all $u \in H^{1}(\Omega)$,

$$
\mu_{1}\left(\int m(x) u^{2}+\oint \rho(x) u^{2}\right) \leqslant \int|\nabla u|^{2}+\int c(x) u^{2}+\oint \sigma(x) u^{2},
$$

where $\mu_{1}>0$ is the least Steklov-Robin eigenvalue for equation (2.1). If equality holds in (2.18), then $u$ is a multiple of an eigenfunction of equation (2.1) corresponding to $\mu_{1}$.

(ii) For every $v \in \bigoplus_{i \leqslant j} E\left(\mu_{i}\right)$, and $w \in \bigoplus_{i \geqslant j+1} E\left(\mu_{i}\right)$, we have that

$$
\|v\|_{(c, \sigma)}^{2} \leqslant \mu_{j}\|v\|_{(m, \rho)}^{2} \quad \text { and } \quad\|w\|_{(c, \sigma)}^{2} \geqslant \mu_{j+1}\|w\|_{(m, \rho)}^{2},
$$

where $E\left(\mu_{i}\right)$ is the $\mu_{i}$-eigenspace and $\bigoplus_{i \leqslant j} E\left(\mu_{i}\right)$ is the span of eigenfunctions associated to eigenvalues up to $\mu_{j}$. 
Proof. If $u=0$, then the inequality (2.18) holds. Otherwise, if $0 \neq u \in H^{1}(\Omega)$, then $u=u_{1}+u_{2}$, where $u_{1} \in H_{(m, \rho)}^{1}$ and $u_{2} \in V_{(m, \rho)}$. Therefore, by the $(c, \sigma)$ orthogonality, and the characterization of $\mu_{1}$ in (2.12), we get that

$$
\begin{aligned}
\left\|u_{1}\right\|_{(c, \sigma)}^{2}+\left\|u_{2}\right\|_{(c, \sigma)}^{2} & \geqslant \mu_{1}\left(\int m(x) u_{1}^{2}+\oint \rho(x) u_{1}^{2}+\int m(x) u_{2}^{2}+\oint \rho(x) u_{2}^{2}\right) \\
& =\mu_{1}\left(\int m(x) u^{2}+\oint \rho(x) u^{2}\right) .
\end{aligned}
$$

Thus, the inequality (2.18) holds. The inequalities (2.19) follow from (2.6)-(2.8).

The following proposition shows the principality of the first eigenvalue $\mu_{1}$.

Proposition 2.3. The first eigenvalue $\mu_{1}$ is simple, and its associated eigenfunction $\varphi_{1}$ is strictly positive (or strictly negative) in $\Omega$.

Proof. We first show that $\varphi_{1}$ does not change sign in $\Omega$. Indeed, suppose it does, and let $\varphi_{1}^{+}=\max \left\{\varphi_{1}, 0\right\}$ and $\varphi_{1}^{-}=\min \left\{\varphi_{1}, 0\right\}$, we know that $\varphi_{1}^{+}$and $\varphi_{1}^{-} \in H^{1}(\Omega)$ (see $[10])$.

By the characterization of $\mu_{1}$ it follows that $\left(\varphi_{1}, \varphi_{1}\right)_{(c, \sigma)}=\mu_{1}\left(\varphi_{1}, \varphi_{1}\right)_{(m, \rho)}$.

Therefore,

$$
\begin{aligned}
0 \leqslant & \left(\varphi_{1}^{+}, \varphi_{1}^{+}\right)_{(c, \sigma)}+\left(\varphi_{1}^{-}, \varphi_{1}^{-}\right)_{(c, \sigma)} \\
& \quad-\mu_{1}\left(\varphi_{1}^{+}, \varphi_{1}^{+}\right)_{(m, \rho)}-\mu_{1}\left(\varphi_{1}^{-}, \varphi_{1}^{-}\right)_{(m, \rho)} \\
= & \left(\varphi_{1}, \varphi_{1}\right)_{(c, \sigma)}-\mu_{1}\left(\varphi_{1}, \varphi_{1}\right)_{(m, \rho)} \\
= & 0 .
\end{aligned}
$$

It follows immediately that $\varphi_{1}^{+}$and $\varphi_{1}^{-}$are also eigenfunctions corresponding to $\mu_{1}$. From $[5,22]$ we get that $\varphi_{1}^{+}>0$ a.e. in $\Omega$ and $\varphi_{1}^{-}<0$ a.e. in $\Omega$, which is impossible. Thus, $\varphi_{1}$ does not change sign in $\Omega$.

Next, we claim that $\mu_{1}$ is simple if and only if $\varphi_{1}$ does not changes sign. Indeed, if $\varphi_{1}$ changes sign, then $\varphi_{1}^{+}$and $\varphi_{1}^{-}$are also eigenfunctions corresponding to $\mu_{1}$ and they are linearly independent. Hence, $\mu_{1}$ is not simple. On the other hand, suppose that $\mu_{1}$ is not simple and let $\varphi$ and $\psi$ be two eigenfunctions corresponding to $\mu_{1}$; they are linearly independent. If $\varphi$ or $\psi$ changes sign, then the claim is proved. Otherwise, supposing without loss of generality that $\varphi$ and $\psi$ are positive, we will prove that there exists $a \in \mathbb{R}$ such that the eigenfunction (corresponding to $\mu_{1}$ ) $\varphi+a \psi$ changes sign. Indeed, suppose that, for all $\alpha \in \mathbb{R}, \varphi+\alpha \psi$ does not change. Let the function $h: \mathbb{R} \rightarrow \mathbb{R}$ be defined by

$$
h(\alpha)=\int \varphi+\alpha \int \psi .
$$

Since $h$ is continuous, there exists $a \in \mathbb{R}$ such that

$$
h(a)=\int \varphi+a \int \psi=0 .
$$

Hence, $\varphi=-a \psi$, which contradicts the fact that $\varphi$ and $\psi$ are linearly independent. Thus, $\varphi+a \psi$ changes sign. The proof is complete. 
REMARK 2.4. Note that if we have smooth data and $\partial \Omega \in C^{2}$ in proposition 2.3, then the eigenfunction $\varphi_{1}(x)>0$ on $\partial \Omega$ as well, by the boundary point lemma (see, for example, $[10])$.

\section{Non-resonance problems}

In this section we take up the nonlinear problem (1.1). We obtain the existence results, which consist of non-resonance with respect to the first Steklov-Robin eigenvalue, and then subsequently with respect to two consecutive higher Steklov-Robin eigenvalues.

ThEorem 3.1 (non-resonance below the first Steklov-Robin eigenvalue). Suppose that the assumptions $\left(\mathrm{C}_{1}\right)-\left(\mathrm{C}_{3^{\prime}}\right)$ are met. Let the potentials

$$
F(x, u)=\int_{0}^{u} f(x, s) \mathrm{d} s \quad \text { and } \quad G(x, u)=\int_{0}^{u} g(x, s) \mathrm{d} s
$$

be such that the following conditions hold.

$\left(\mathrm{C}_{4}\right)$ There exist $\lambda, \mu \in \mathbb{R}$ such that

$$
\limsup _{|u| \rightarrow \infty} \frac{2 F(x, u)}{u^{2}} \leqslant \lambda m(x) \quad \text { and } \quad \limsup _{|u| \rightarrow \infty} \frac{2 G(x, u)}{u^{2}} \leqslant \mu \rho(x)
$$

uniformly for $x \in \bar{\Omega}$, where

$$
\max (\lambda, \mu)<\mu_{1} .
$$

Then, equation (1.1) has at least one solution $u \in H^{1}(\Omega)$.

In the next result, we are concerned with the case of non-resonance between two consecutive Steklov-Robin eigenvalues. We impose conditions on the asymptotic behaviour of the nonlinearities $f(x, u)$ and $g(x, u)$. (These conditions imply similar ones on the asymptotic behaviour of the potentials $F(x, u)$ and $G(x, u)$.)

Theorem 3.2 (non-resonance between consecutive Steklov-Robin eigenvalues). Suppose that assumptions $\left(\mathrm{C}_{1}\right)-\left(\mathrm{C}_{3^{\prime}}\right)$ are met, and that the following conditions hold.

$\left(\mathrm{C}_{5}\right)$ There exist constants $a, b, \alpha, \beta \in \mathbb{R}$ such that

$$
\alpha m(x) \leqslant \liminf _{|u| \rightarrow \infty} \frac{f(x, u)}{u} \leqslant \limsup _{|u| \rightarrow \infty} \frac{f(x, u)}{u} \leqslant \beta m(x)
$$

and

$$
a \rho(x) \leqslant \liminf _{|u| \rightarrow \infty} \frac{g(x, u)}{u} \leqslant \limsup _{|u| \rightarrow \infty} \frac{g(x, u)}{u} \leqslant b \rho(x),
$$

uniformly for $x \in \bar{\Omega}$, where

$$
\mu_{j}<\min (a, \alpha) \leqslant \max (b, \beta)<\mu_{j+1} .
$$

Then, equation (1.1) has at least one solution $u \in H^{1}(\Omega)$. 
Unlike previous results in the literature, what sets our existence results apart here is that we compare both the nonlinearities in the differential equation and on the boundary with higher eigenvalues of the generalized Steklov-Robin spectrum of problem (1.2) in which the spectral parameter is both in the differential equation and on the boundary.

\section{Proofs of non-resonance theorems}

Our approach to problem (1.1) is variational and uses the saddle-point theorem and its variants proved in [21]. The functional associated with our problem (1.1) is

$$
I(u)=\frac{1}{2}\left[\int|\nabla u|^{2}+\int c(x) u^{2}+\oint \sigma(x) u^{2}\right]-\int F(x, u)-\oint G(x, u) .
$$

It follows from assumptions $\left(\mathrm{C}_{1}\right)-\left(\mathrm{C}_{3^{\prime}}\right)$ that this functional is of class $C^{1}$ in $H^{1}(\Omega)$ with

$$
\begin{aligned}
I^{\prime}(u) v=\int \nabla u \nabla v+\int c(x) u v+\oint \sigma(x) u v & \\
& -\int f(x, u) v-\oint g(x, u) v \text { for every } v \in H^{1}(\Omega)
\end{aligned}
$$

(see, for example, [17] for the proofs of this claim). Clearly, the critical points of $I$ are precisely the (weak) solutions of equation (1.1).

Proof of theorem 3.1. Observe that condition $\left(\mathrm{C}_{4}\right)$ implies that, for every $\epsilon>0$, there is $r=r(\epsilon)>0$ such that

$$
\frac{2 G(x, u)}{u^{2}} \leqslant \mu \rho(x)+\epsilon \text { and } \quad \frac{2 F(x, u)}{u^{2}} \leqslant \lambda m(x)+\epsilon
$$

for all $x \in \bar{\Omega}$ and all $u \in \mathbb{R}$ with $|u|>r$. Combining (4.1) with $\left(\mathrm{C}_{3}\right)$ and $\left(\mathrm{C}_{3^{\prime}}\right)$, there exists a constant $M_{\epsilon}>0$ such that

$$
\left.\begin{array}{c}
G(x, u) \leqslant \frac{1}{2}(\mu \rho(x)+\epsilon) u^{2}+M_{\epsilon}, \\
F(x, u) \leqslant \frac{1}{2}(\lambda m(x)+\epsilon) u^{2}+M_{\epsilon}, \\
\forall x \in \bar{\Omega}, \forall u \in \mathbb{R},
\end{array}\right\}
$$

To prove that equation (1.1) has at least one solution, it suffices, according to [21, p. 8, theorem 2.7], to show that the functional $I$ is bounded below and that it satisfies the Palais-Smale condition (that is, any sequence $\left\{u_{n}\right\}$ in $H^{1}(\Omega)$ such that $\left\{I\left(u_{n}\right)\right\}$ is bounded, and $\lim _{n \rightarrow \infty} I^{\prime}\left(u_{n}\right)=0$, be precompact). Under the assumptions of theorem 3.1, we shall prove that the functional $I$ is coercive on $H^{1}(\Omega)$; that is,

$$
I(u) \rightarrow \infty \quad \text { as }\|u\|_{(c, \sigma)} \rightarrow \infty .
$$

Then it will follow that $I$ is bounded below and that it satisfies the Palais-Smale. 
From (4.2) we obtain that

$$
\begin{aligned}
I(u) & \geqslant \frac{1}{2}\|u\|_{(c, \sigma)}^{2}-\frac{1}{2} \int(\lambda m(x)+\epsilon) u^{2}-\frac{1}{2} \oint(\mu \rho(x)+\epsilon) u^{2}-C \\
& \geqslant \frac{1}{2}\|u\|_{(c, \sigma)}^{2}-\frac{1}{2} \kappa\left[\int m(x) u^{2}+\oint \rho(x) u^{2}\right]-\frac{1}{2} \epsilon\left[\int u^{2}+\oint u^{2}\right]-C,
\end{aligned}
$$

where $\kappa=\max (\lambda, \mu)$. Using the inequality (2.18), the Sobolev embedding of $H^{1}(\Omega)$ into $L^{2}(\Omega)$, and the continuity of the trace operator from $H^{1}(\Omega)$ into $L^{2}(\partial \Omega)$, we have that

$$
I(u) \geqslant \frac{1}{2}\left(1-\frac{\kappa}{\mu_{1}}-\epsilon K\right)\|u\|_{(c, \sigma)}^{2}-C
$$

Since $\kappa<\mu_{1}$, it follows that $1-\left(\kappa / \mu_{1}\right)-\epsilon K>0$ for sufficiently small $\epsilon$. Therefore, we get that $I(u) \rightarrow \infty$ as $\|u\|_{(c, \sigma)} \rightarrow \infty$. Thus, $I$ is coercive. It follows immediately that $I$ is bounded from below and that it satisfies the Palais-Smale condition. From [21, theorem 2.7, chapter 2], we get that $I$ has a critical point $u \in H^{1}(\Omega)$, that is, $I^{\prime}(u)=0$. Thus, equation (1.1) has at least one solution. The proof is complete.

Proof of theorem 3.2. Under the assumptions of theorem 3.2 we need to show that the conditions of the saddle-point theorem are fulfilled. First we decompose the space $H^{1}(\Omega)$ as

$$
H^{1}(\Omega)=W \oplus_{(c, \sigma)} X
$$

where

$$
W=\bigoplus_{i \leqslant j} E\left(\mu_{i}\right), \quad X=Y \oplus_{(c, \sigma)} V_{(m, \rho)}(\Omega) \text { with } Y=\overline{\bigoplus_{i \geqslant j+1} E\left(\mu_{i}\right)} .
$$

We need to prove that there exists a constant $r>0$ such that

$$
\sup _{\partial D} I<\inf _{X} I
$$

where $D=\left\{v \in W:\|u\|_{(c, \sigma)} \leqslant r\right\}$. Assuming that this is the case, and that the Palais-Smale condition is satisfied, we deduce by the saddle-point theorem [21] that $I$ has a critical point. Therefore, equation (1.1) has at least one solution.

Now we shall show that the functional $\left.I\right|_{X}$ and $\left.(-I)\right|_{W}$ are coercive, which would imply that (4.5) is satisfied by choosing $r>0$ sufficiently large.

Observe that condition $\left(\mathrm{C}_{5}\right)$ implies a similar condition on the potential $G$; that is, there exist constants again called $a, b, \alpha, \beta \in \mathbb{R}$ such that, for all $x \in \bar{\Omega}$,

$$
\begin{gathered}
a \rho(x) \leqslant \liminf _{|u| \rightarrow \infty} \frac{2 G(x, u)}{u^{2}} \leqslant \limsup _{|u| \rightarrow \infty} \frac{2 G(x, u)}{u^{2}} \leqslant b \rho(x), \\
\alpha m(x) \leqslant \liminf _{|u| \rightarrow \infty} \frac{2 F(x, u)}{u^{2}} \leqslant \limsup _{|u| \rightarrow \infty} \frac{2 F(x, u)}{u^{2}} \leqslant \beta m(x) .
\end{gathered}
$$


Combining $\left(\mathrm{C}_{3}\right),\left(\mathrm{C}_{3^{\prime}}\right),(4.6)$ and $(4.7)$, we get that, for all $\epsilon>0$, all $x \in \bar{\Omega}$ and all $u \in \mathbb{R}$

$$
\begin{gathered}
\frac{1}{2}(a \rho(x)-\epsilon) u^{2}-C \leqslant G(x, u) \leqslant \frac{1}{2}(b \rho(x)+\epsilon) u^{2}+C, \\
\frac{1}{2}(\alpha m(x)-\epsilon) u^{2}-C \leqslant F(x, u) \leqslant \frac{1}{2}(\beta m(x)+\epsilon) u^{2}+C,
\end{gathered}
$$

where $C$ is a positive constant.

On the one hand, for every $u \in W$, we have that

$$
\begin{aligned}
I(u) & \leqslant \frac{1}{2}\|u\|_{(c, \sigma)}^{2}-\frac{1}{2} \int(\alpha m(x)-\epsilon) u^{2}-\frac{1}{2} \oint(a \rho(x)-\epsilon) u^{2}+\tilde{C} \\
& \leqslant \frac{1}{2}\|u\|_{(c, \sigma)}^{2}-\frac{1}{2} \kappa_{1}\left[\int m(x) u^{2}+\oint \rho(x) u^{2}\right]+\frac{1}{2} \epsilon\left[\int u^{2}+\oint u^{2}\right]+C \\
& \leqslant \frac{1}{2}\|u\|_{(c, \sigma)}^{2}-\frac{1}{2} \kappa_{1}\left[\int m(x) u^{2}+\oint \rho(x) u^{2}\right]+\frac{1}{2} \epsilon K\|u\|_{(c, \sigma)}^{2}+C
\end{aligned}
$$

where $\kappa_{1}=\min (a, \alpha)$. It follows from (2.19) that

$$
\begin{aligned}
I(u) & \leqslant \frac{1}{2}\|u\|_{(c, \sigma)}^{2}-\frac{1}{2} \frac{\kappa_{1}}{\mu_{j}}\|u\|_{(c, \sigma)}^{2}+\frac{1}{2} \epsilon K\|u\|_{(c, \sigma)}^{2}+C \\
& =\frac{1}{2}\left(1-\frac{\kappa_{1}}{\mu_{j}}+\epsilon K\right)\|u\|_{(c, \sigma)}^{2}+C
\end{aligned}
$$

Since $\kappa_{1}>\mu_{j}$, it follows that $1-\left(\kappa_{1} / \mu_{j}\right)+\epsilon K<0$ for $\epsilon$ sufficiently small, and we obtain that

$$
I(u) \rightarrow-\infty \quad \text { as }\|u\|_{(c, \sigma)} \rightarrow \infty
$$

On the other hand, for every $u \in X$, it follows from (4.4) that $u=v+\bar{u}$, where $v \in V_{(m, \rho)}(\Omega)$ and $\bar{u} \in Y$. Taking into account the $(c, \sigma)$-orthogonality of $\bar{u}$ and $v$ in $H^{1}(\Omega)$, we have that

$$
\begin{aligned}
I(u) & \geqslant \frac{1}{2}\|v\|_{(c, \sigma)}^{2}+\frac{1}{2}\|\bar{u}\|_{(c, \sigma)}^{2}-\frac{1}{2} \int(\beta m(x)+\epsilon) u^{2}-\frac{1}{2} \oint(b \rho(x)+\epsilon) u^{2}-\tilde{C} \\
& \geqslant \frac{1}{2}\|v\|_{(c, \sigma)}^{2}+\frac{1}{2}\|\bar{u}\|_{(c, \sigma)}^{2}-\frac{1}{2} \kappa_{2}\left[\int m(x) u^{2}+\oint \rho(x) u^{2}\right]-\frac{1}{2} \epsilon K\|u\|_{(c, \sigma)}^{2}-\tilde{C},
\end{aligned}
$$

where $\kappa_{2}=\max (b, \beta)$. Since $v \in V_{(m, \rho)}(\Omega)$ we have that

$$
\int m(x) v^{2}+\oint \rho(x) v^{2}=0 \quad \text { and } \quad \int m(x) v \bar{u}+\oint \rho(x) v \bar{u}=0 .
$$

Therefore,

$$
I(u) \geqslant \frac{1}{2}\|v\|_{(c, \sigma)}^{2}+\frac{1}{2}\|\bar{u}\|_{(c, \sigma)}^{2}-\frac{1}{2} \kappa_{2}\|\bar{u}\|_{(m, \rho)}-\frac{1}{2} \epsilon K\|u\|_{(c, \sigma)}^{2}-\tilde{C} .
$$


It follows from (2.19) that

$$
\begin{aligned}
I(u) & \geqslant \frac{1}{2}\|v\|_{(c, \sigma)}^{2}+\frac{1}{2}\left(1-\frac{\kappa_{2}}{\mu_{j+1}}\right)\|\bar{u}\|_{(c, \sigma)}-\frac{1}{2} \epsilon K\|u\|_{(c, \sigma)}^{2}-\tilde{C} \\
& \geqslant \frac{1}{2}\left(1-\frac{\kappa_{2}}{\mu_{j+1}}\right)\left(\|\bar{u}\|_{(c, \sigma)}+\|v\|_{(c, \sigma)}^{2}\right)-\frac{1}{2} \epsilon K\|u\|_{(c, \sigma)}^{2}-\tilde{C} \\
& =\frac{1}{2}\left(1-\frac{\kappa_{2}}{\mu_{j+1}}-\epsilon K\right)\|u\|_{(c, \sigma)}^{2}-\tilde{C} .
\end{aligned}
$$

Since $\kappa_{2}<\mu_{j+1}$, it follows that $1-\left(\kappa_{2} / \mu_{j+1}\right)-\epsilon K>0$ for $\epsilon$ sufficiently small, and we obtain that

$$
I(u) \rightarrow \infty \quad \text { as }\|u\|_{(c, \sigma)} \rightarrow \infty .
$$

Therefore, $I$ is coercive on $X, \vartheta=\inf _{X} I(u)>-\infty$ is attained. Now, we take $\delta<\vartheta$. By (4.10) there exists $r>0$ such that $I(u) \leqslant \delta$ for all $u \in W$ with $\|u\|_{(c, \sigma)} \geqslant r$. Therefore, (4.5) holds.

It remains to prove that the functional $I$ satisfies the Palais-Smale condition. It suffices to show that, for any sequence $\left\{u_{n}\right\}$ in $H^{1}(\Omega)$ such that $\left\{I\left(u_{n}\right)\right\}$ is bounded and $\lim _{n \rightarrow \infty} I^{\prime}\left(u_{n}\right)=0$, we have that $\left\{u_{n}\right\}$ is bounded (see, for example, $[17,21]$ ).

Note that condition $\left(\mathrm{C}_{5}\right)$ implies that, for every $\epsilon>0$, there exists $r>0$ such that, for $|u| \geqslant r$,

$$
a \rho(x)-\epsilon \leqslant \frac{g(x, u)}{u} \leqslant b \rho(x)+\epsilon \text { for all } x \in \bar{\Omega} .
$$

Let us define $\tilde{\gamma}: \bar{\Omega} \times \mathbb{R} \rightarrow \mathbb{R}$ by

$$
\tilde{\gamma}(x, u)= \begin{cases}\frac{g(x, u)}{u} & \text { for }|u| \geqslant r, \\ \frac{g(x, r)+g(x,-r)}{2 r^{2}} u+\frac{g(x, r)-g(x,-r)}{2 r} & \text { for }|u|<r .\end{cases}
$$

The function $\tilde{\gamma}$ is continuous in $\bar{\Omega} \times \mathbb{R}$ since $g$ is. Moreover, by (4.12) one has

$$
a \rho(x)-\epsilon \leqslant \tilde{\gamma}(x, u) \leqslant b \rho(x)+\epsilon \text { for all } u \in \mathbb{R} \text { and for all } x \in \bar{\Omega} .
$$

Define $\tilde{h}: \bar{\Omega} \times \mathbb{R} \rightarrow \mathbb{R}$ by

$$
\tilde{h}(x, u)=g(x, u)-\tilde{\gamma}(x, u) u .
$$

Then it follows from the continuity of $g$ and $\gamma$ that $|\tilde{h}(x, u)| \leqslant K$ for all $(x, u) \in$ $\bar{\Omega} \times \mathbb{R}$, where $K>0$ is a constant.

Using a similar decomposition for the function $f$, we get that

$$
\bar{h}(x, u)=f(x, u)-\bar{\gamma}(x, u) u,
$$

where $\bar{\gamma}$ and $\bar{h}$ satisfy

$$
\alpha m(x)-\epsilon \leqslant \bar{\gamma}(x, u) \leqslant \beta m(x)+\epsilon \quad \text { and } \quad|\bar{h}(x, u)| \leqslant K,
$$

for all $(x, u) \in \bar{\Omega} \times \mathbb{R}$, where $K>0$ is a constant. 
Now, let $\left\{u_{n}\right\} \subset H^{1}(\Omega)$ be such that $\left\{I\left(u_{n}\right)\right\}$ is bounded and $\lim _{m \rightarrow \infty} I^{\prime}\left(u_{n}\right)=0$. Since $u_{n} \in H^{1}(\Omega)$, we have that $u_{n}=w_{n}+x_{n}$, where $w_{n} \in W$ and $x_{n} \in X$ with $x_{n}=v_{n}+y_{n}$, where $v_{n} \in V_{(m, \rho)}$ and $y_{n} \in Y$.

Since $\lim _{n \rightarrow \infty} I^{\prime}\left(u_{n}\right)=0$, it follows that, for every $\epsilon>0$, there exists $N>0$ such that, for all $n \geqslant N$,

$$
\sup _{\varphi \neq 0} \frac{\left|I^{\prime}\left(u_{n}\right) \varphi\right|}{\|\varphi\|_{(c, \sigma)}}<\epsilon
$$

Set $\varphi=x_{n}-w_{n}$ for $n$ large. Then $I^{\prime}\left(u_{n}\right)\left(x_{n}-w_{n}\right)<\epsilon\left\|x_{n}-w_{n}\right\|_{(c, \sigma)}$. Taking into account the $(c, \sigma)$-orthogonality of $x_{n}$ and $w_{n}$ in $H^{1}(\Omega),(4.14)$ and (4.15), we obtain from the definition of $I^{\prime}$ that

$$
\begin{aligned}
& \left\|x_{n}\right\|_{(c, \sigma)}^{2}-\left\|w_{n}\right\|_{(c, \sigma)}^{2}-\int \bar{\gamma}\left(x, u_{n}\right) x_{n}^{2}+\int \bar{\gamma}\left(x, u_{n}\right) w_{n}^{2} \\
& -\oint \tilde{\gamma}\left(x, u_{n}\right) x_{n}^{2}+\oint \tilde{\gamma}\left(x, u_{n}\right) w_{n}^{2} \\
& <\epsilon\left(\left\|x_{n}\right\|_{(c, \sigma)}+\left\|w_{n}\right\|_{(c, \sigma)}\right)+\int \bar{h}\left(x, u_{n}\right) x_{n}-\int \bar{h}\left(x, u_{n}\right) w_{n} \\
& \quad+\oint \tilde{h}\left(x, u_{n}\right) x_{n}-\oint \tilde{h}\left(x, u_{n}\right) w_{n} .
\end{aligned}
$$

By using (4.13)-(4.16), the continuity of the trace operator, and the fact that $\min (a, \alpha)=\kappa_{1}, \max (b, \beta)=\kappa_{2}$ and $x_{n}=v_{n}+y_{n}$ with $v_{n} \in V_{(m, \rho)}$ and $y_{n} \in Y$, we obtain that

$$
\begin{aligned}
\left\|v_{n}\right\|_{(c, \sigma)}^{2}+ & \left\|y_{n}\right\|_{(c, \sigma)}^{2}-\left\|w_{n}\right\|_{(c, \sigma)}^{2}-\kappa_{2}\left\|y_{n}\right\|_{(m, \rho)}^{2}+\kappa_{1}\left\|w_{n}\right\|_{(m, \rho)}^{2} \\
& -\epsilon C\left(\left\|x_{n}\right\|_{(c, \sigma)}^{2}+\left\|w_{n}\right\|_{(c, \sigma)}^{2}\right)<(\epsilon+\tilde{K})\left(\left\|x_{n}\right\|_{(c, \sigma)}+\left\|w_{n}\right\|_{(c, \sigma)}\right) .
\end{aligned}
$$

It follows from (2.19) that

$$
\begin{aligned}
\left\|v_{n}\right\|_{(c, \sigma)}^{2}+ & \left\|y_{n}\right\|_{(c, \sigma)}^{2}-\left\|w_{n}\right\|_{(c, \sigma)}^{2}-\kappa_{2} \frac{1}{\mu_{j+1}}\left\|y_{n}\right\|_{(c, \sigma)}^{2}+\kappa_{1} \frac{1}{\mu_{j}}\left\|w_{n}\right\|_{(c, \sigma)}^{2} \\
& -\epsilon C\left(\left\|x_{n}\right\|_{(c, \sigma)}^{2}+\left\|w_{n}\right\|_{(c, \sigma)}^{2}\right)<(\epsilon+\tilde{K})\left(\left\|x_{n}\right\|_{(c, \sigma)}+\left\|w_{n}\right\|_{(c, \sigma)}\right) .
\end{aligned}
$$

Therefore,

$$
\begin{aligned}
\left\|v_{n}\right\|_{(c, \sigma)}^{2}+ & \left(1-\frac{\kappa_{2}}{\mu_{j+1}}\right)\left\|y_{n}\right\|_{(c, \sigma)}^{2}+\left(\frac{\kappa_{1}}{\mu_{j}}-1\right)\left\|w_{n}\right\|_{(c, \sigma)}^{2} \\
& -\epsilon C\left(\left\|x_{n}\right\|_{(c, \sigma)}^{2}+\left\|w_{n}\right\|_{(c, \sigma)}^{2}\right)<(\epsilon+\tilde{K})\left(\left\|x_{n}\right\|_{(c, \sigma)}+\left\|w_{n}\right\|_{(c, \sigma)}\right) .
\end{aligned}
$$

Hence, for $\epsilon$ sufficiently small,

$$
\delta\left(\left\|x_{n}\right\|_{(c, \sigma)}^{2}+\left\|w_{n}\right\|_{(c, \sigma)}^{2}\right)<(\epsilon+\tilde{K})\left(\left\|x_{n}\right\|_{(c, \sigma)}+\left\|w_{n}\right\|_{(c, \sigma)}\right),
$$

where $\delta=\min \left\{1,\left(\kappa_{1} / \mu_{j}\right)-1,1-\left(\kappa_{2} / \mu_{j+1}\right)\right\}$. Therefore,

$$
\left\|u_{n}\right\|_{c}^{2}<\tilde{K}_{0}\left\|u_{n}\right\|_{c}
$$

which implies that $\left\{u_{n}\right\}$ is bounded in $H^{1}(\Omega)$. Thus, $I$ satisfies the Palais-Smale condition. 
REMARK 4.1.

(i) Note that, in theorem 3.1, we impose conditions on the potential of the nonlinearities rather than on the nonlinearities themselves, as was the case in previous papers. In this case, we do not require a (one-sided) linear growth condition on $f$ and $g$ as was done in $[1,11,13,14]$. Nonlinearities may also be superlinear at infinity. For illustration purposes, take $n=3$ and consider the nonlinearities

$$
\begin{aligned}
& f(x, u)= \begin{cases}u^{4}+A(x) & \text { if } u<0, \\
-u^{4}+A(x) & \text { if } u \geqslant 0,\end{cases} \\
& g(x, u)= \begin{cases}u^{2}+B(x) & \text { if } u<0, \\
-u^{2}+B(x) & \text { if } u \geqslant 0,\end{cases}
\end{aligned}
$$

where $A(x), B(x) \in C(\bar{\Omega})$. We see that the nonlinearities $f(x, u)$ and $g(x, u)$ satisfy conditions $\left(\mathrm{C}_{3}\right)$ and $\left(\mathrm{C}_{3^{\prime}}\right)$, and that the potentials $F(x, u)$ and $G(x, u)$ generated by $f$ and $g$ satisfy condition $\left(\mathrm{C}_{4}\right)$.

(ii) Because of the non-resonance condition $\left(\mathrm{C}_{5}\right)$, the nonlinearities considered in theorem 3.2 are at most linear at infinity.

REMARK 4.2. Our results remain valid if one replaces the functions $f(x, u)$ and $g(x, u)$ with $f(x, u)+A(x)$ and $g(x, u)+B(x)$ respectively, where $A \in L^{2}(\Omega)$ and $B \in L^{2}(\partial \Omega)$.

If $f(x, 0)=0$ for $x \in \Omega$ and $g(x, 0)=0$ for $x \in \partial \Omega$, then generally additional conditions on the behaviour of the ratios $f(x, u) / u$ and $g(x, u) / u$ near zero are imposed to ensure the existence of non-trivial solutions of problem (1.1) (see, for example, [11] for the case near the first eigenvalue).

REMARK 4.3. Our non-resonance results remain valid if one considers nonlinear equations with a more general linear part (in divergence form) with variable coefficients.

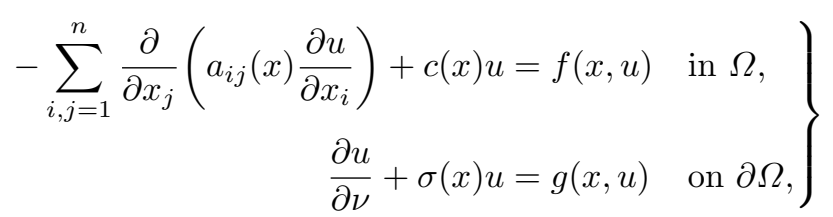

where $\sigma \in L^{\infty}(\partial \Omega)$ with $\sigma(x) \geqslant 0$ a.e. on $\partial \Omega$, and $\partial / \partial \nu:=\nu \cdot A \nabla$ is the (unit) outward conormal derivative. The matrix $A(x):=\left(a_{i j}(x)\right)$ is symmetric with $a_{i j} \in$ $L^{\infty}(\Omega)$ such that there is a constant $\gamma>0$ such that, for all $\xi \in \mathbb{R}^{n}$,

$$
\langle A(x) \xi, \xi\rangle \geqslant \gamma|\xi|^{2} \quad \text { a.e. on } \Omega \text {. }
$$

Remark 4.4. Note that, when $c \equiv 0$ and $\sigma \equiv 0$, the first (generalized) SteklovRobin eigenvalue $\mu_{1}=0$ with the corresponding eigenfunction constant (see, for example, $[2,6,20])$.

\section{Acknowledgements}

The author wishes to thank the referee for a careful reading of the paper and for pointing out [11]. 


\section{References}

1 H. Amann. Nonlinear elliptic equations with nonlinear boundary conditions. In New developments in differential equations, Proc. 2nd Schrveningen Conf. Diff. Eqns, North-Holland Mathematics Studies, vol. 21, pp. 43-64 (Amsterdam: North-Holland, 1976).

2 G. Auchmuty. Steklov eigenproblems and the representation of solutions of elliptic boundary value problems. Numer. Func. Analysis Optim. 25 (2004), 321-348.

3 C. Bandle. Isoperimetric inequalities and applications (London: Pitman, 1980).

4 A. Castro. Semilinear equations with discrete spectrum. Contemp. Math. 347 (2004), 1-16.

5 M. Chicco. Principio di massimo generalizzato e valutazione del primo autovalore per problemi ellittici del secondo ordine di tipo variazionale. Annali Mat. Pura Appl. 87 (1970), $1-9$.

6 J. M. Cushing. Nonlinear Steklov problems on the unit circle. J. Math. Analysis Applic. 38 (1972), 766-783.

7 D. G. de Figueiredo. Positive solutions of semilinear elliptic problems. In Proceedings of the 1st Latin American school of differential equations, São Paulo, Brazil, June 29-July 17, Lecture Notes in Mathematics, vol. 957, pp. 34-87 (Springer, 1982).

8 D. G. de Figueiredo and J. P. Gossez. Conditions de non-résonance pour certains problèmes élliptiques semi-lineaires. C. R. Acad. Sci. Paris Sér. I 302 (1986), 543-545.

9 J. F. Escobar. A comparison theorem for the first non-zero Steklov eigenvalue. J. Funct. Analysis 178 (2000), 143-155.

10 L. C. Evans. Partial differential equations (Providence, RI: American Mathematical Society, 1998).

11 J. García-Melián, J. D. Rossi and J. Sabina de Lis. Existence and uniqueness of positive solutions to elliptic problems with sublinear mixed boundary conditions. Commun. Contemp. Math. 11 (2009), 585-613.

12 R. Iannacci and M. Nkashama. Unbounded perturbations of forced second order ordinary differential equations at resonance. J. Diff. Eqns 69 (1986), 289-309.

13 K. Klingelhöfer. Nonlinear harmonic boundary value problems. I. Arch. Ration. Mech. Analysis 31 (1968), 364-371.

14 K. Klingelhöfer. Nonlinear boundary value problems with simple eigenvalue of the linear part. Arch. Ration. Mech. Analysis 37 (1970), 381-398.

15 A. Kufner, O. John and S. Fučík. Function spaces (Leyden: Noordhoff, 1977).

16 A. Manes and A. M. Micheletti. Un'estensione della teoria variazionale classica degli autovalori per operatri ellitici del secondo ordine. Boll. UMI A 7 (1973), 285-301.

17 N. Mavinga and M. N. Nkashama. Steklov-Neumann eigenproblems and nonlinear elliptic equations with nonlinear boundary conditions. J. Diff. Eqns 248 (2010), 1212-1229.

18 J. Mawhin and K. Schmitt. Corrigendum: upper and lower solutions and semilinear second order elliptic equations with non-linear boundary conditions. Proc. R. Soc. Edinb. A 100 (1985), 361.

19 J. Nečas. Les methodes directes en théorie des equations elliptiques (Paris: Masson, 1967).

20 M. N. Nkashama. Saddle point theorem and nonlinear scalar Neumann boundary value problems. J. Austral. Math. Soc. A 55 (1993), 386-402.

21 P. H. Rabinowitz. Minimax methods in critical point theory with application to differential equations, CBMS Regional Conf. Ser. Math. no. 65 (Providence, RI: American Mathematical Society, 1986).

22 G. Stampacchia. Le problème de Dirichlet pour les équations elliptiques du second ordre à coefficients discontinus. Annales Inst. Fourier 15 (1965), 189-258.

23 M. W. Steklov. Sur les problèmes fundamentaux de la physique mathématique. Annali Scuola Norm. Sup. Pisa 19 (1902), 455-490.

(Issued 17 February 2012) 